\title{
Conocimiento y percepciones de los estudiantes del aula virtual en la Universidad Nacional de Pilar
}

\author{
Knowledge and perceptions of students in the Virtual \\ Classroom at the Universidad Nacional de Pilar
}

\author{
Dominga Elsa Velázquez - Néstor Samuel Torres Velázquez \\ PARAGUAY
}

\begin{abstract}
Resumen: el uso de las Tecnologías de la Información y la Comunicación (TIC) en el contexto educativo requiere de un cambio en las capacidades relacionadas con el manejo de los Entornos Virtuales de Aprendizaje (EVA). Este trabajo estudia el nivel de conocimiento y las percepciones de los estudiantes respecto al uso de los EVA aplicado al Aula Virtual en la plataforma Moodle. El tipo de estudio fue descriptivo, cuantitativo y transversal. Se aplicó un cuestionario on-line a 347 estudiantes. Se encontró que tan solo el $40 \%$ posee un dominio elevado del uso de las aplicaciones académicas utilizadas, la señal de internet es muy escasa y hay problemas para la compra de uso de datos, entre otros. Falta que docentes y alumnos se capaciten en el uso de los EVA.
\end{abstract}

Palabras clave: tecnología de la información y comunicación (TIC), e-learning; estudiante; enseñanza superior.

\begin{abstract}
The use of Information and Communication Technologies (ICTs) in the educational context requires a change in the capacities related to the management of Virtual Learning Environments (EVA). The purpose of this work seeks to know the level of knowledge and perceptions of students regarding the use of EVA applied to the Virtual Classroom on the Moodle platform. The type of study was descriptive, quantitative, and cross-sectional. An online questionnaire was applied to 347 students. The study found that only $40 \%$ have a high command using the academic applications evaluated, the internet signal is very scarce, and there are problems for the purchase of data plans, among others. Both teachers and students must be trained in the use of Virtual learning environments.
\end{abstract}

Keywords: information and communication technology (TIC); e-learning; student; higher education.

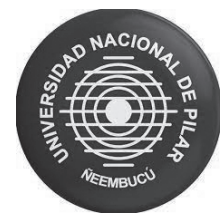

Dominga Elsa Velázquez es Licenciada en Ciencias de la Educación, Magíster en Ciencias de la Educación con énfasis en Investigación Científica y Doctora en Educación. Docente de la Facultad de Ciencias Contables Administrativas y Económicas y de la Facultad de Ciencias Agropecuarias y Desarrollo Rural de la Universidad Nacional de Pilar. Par evaluadora y Docente investigadora, integrante del Grupo de Investigación de la UNP.

Contacto: domingaelsa@gmail.com orcid.org/0000-0001-5742-8882 


\section{Conhecimentos e percepções dos alunos da Sala de Aula Virtual da Universidade Nacional do Pilar}

Resumo: o uso de Tecnologias de Informação e Comunicação (TIC) requer uma mudança nas capacidades relacionadas à gestão de Ambientes Virtuais de Aprendizagem (EVA). O objetivo deste trabalho visa conhecer o nível de conhecimento e percepção dos alunos quanto à utilização do EVA aplicado à Sala de Aula Virtual na plataforma MOODLE. O tipo de estudo foi descritivo, quantitativo e transversal. Um questionário online foi aplicado a 347 alunos. Verificou-se que apenas $40 \%$ possuem alto domínio do uso dos aplicativos acadêmicos utilizados; que o sinal de internet é muito escasso, o problema para a compra de uso de dados, entre outros. Falta que professores e alunos sejam treinados no uso de Ambientes Virtuais de Aprendizagem.

Palavras-chave: tecnologia da informação e comunicação (TIC); e-learning; estudante; ensino superior.

\section{Introducción}

a idea de Nuevas Tecnologías de la Información y la Comunicación (NTIC) es un concepto que ha surgido con la sociedad de la información, que hace alusión a la velocidad con que estas llegan a todo el mundo; en ese sentido, existen diversos autores que definen este concepto, en este trabajo consideramos el referido por Castells (1998) citado por Benítez (2007), quien sostiene que las NTIC constituyen:

un conjunto de tecnologías desarrolladas en el campo de la microelectrónica, la informática, las telecomunicaciones, la televisión y la radio, la optoelectrónica y su conjunto de desarrollos y aplicaciones aquellas herramientas y programas que tratan, administran, transmiten y comparten la información mediante soportes tecnológicos. (p. 67)

Específicamente en la situación en la que se enmarca este trabajo, consideramos que las NTIC comprenden las distintas herramientas utilizadas hoy día en el ámbito académico, tales como los ordenadores, las redes de información, los entornos virtuales, que incluyen la interfaz que permite una comunicación interactiva entre docentes y alumnos, así como garantizar el desarrollo del proceso educativo.

Las Tecnologías de la Información y Comunicación (TIC) se han ido incorporando de manera vertiginosa desde la década de los 90 a todos los

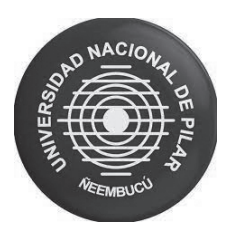

Néstor Samuel Torres Velázquez es Docente de la Facultad de Ciencias Contables, Administrativas y Económicas de la Universidad Nacional de Pilar. Docente de la Facultad Politécnica y Facultad de Ingeniería de la Universidad Nacional de Asunción. Integrante del Comité de autoevaluación de la Politécnica de la UNA y del grupo de desarrollo de aplicaciones COVIDTracker y AlertCOVID-19 del CONACYT.

Contacto: storres@dont.com.py 
campos del saber humano. Este hecho ha representado un reto fundamental para las instituciones de educación superior, al punto que a la sociedad de finales del siglo XX e inicios del siglo XXI se la ha denominado "Sociedad del Conocimiento" o "Sociedad de la Información". Estas tecnologías se han convertido en un instrumento indispensable para la lucha contra la pobreza, prácticamente un requisito para el desarrollo (Ministerio de Ciencia y Tecnología de España, s.f.).

Prácticamente no hay un solo ámbito de la vida humana que no se haya visto impactado por este desarrollo: la salud, las finanzas, los mercados laborales, las comunicaciones, el gobierno, la productividad industrial, etc. El conocimiento se multiplica más rápido que nunca antes y se distribuye de manera casi instantánea. El mundo se ha vuelto un lugar más pequeño e interconectado (UNESCO, 2013). No obstante, esta situación hoy día constituye todo un reto para los países subdesarrollados. Esta realidad también afecta al Paraguay, si se considera que el Presidente de la República ha sacado una Resolución de confinamiento a causa de la pandemia ocasionada por el virus SARS-CoV-2.

Asimismo, el Consejo Nacional de la Educación Superior (CONES) se ha pronunciado mediante la Resolución CE-CONES 04 de 2020: "Que establece la Facultad de las Instituciones de educación superior para aplicar herramientas digitales de enseñanza-aprendizaje en el marco de la emergencia sanitaria covid-19 dispuestas por las autoridades nacionales"; esta resolución ha sido ampliada para el primer semestre del año 2021, mediante la Resolución 57 de 2020, que extiende esta modalidad de aprendizaje (Consejo Nacional de Educación Superior, 2020). Estos hechos, han generado una modificación en la manera de encarar el proceso de enseñanza-aprendizaje para dar continuidad al mismo en la educación superior.

La Universidad Nacional de Pilar (UNP), en ese mismo contexto, se ha visto en la necesidad de implementar nuevas maneras de continuar con el proceso educativo; por ello, mediante la Resolución 114 del 19 de marzo de 2020, proveniente del Consejo Superior Universitario de la UNP, se ha gestionado el uso del aula virtual Moodle en todas las unidades académicas. Este trabajo se plantea ante la necesidad de conocer en qué medida los estudiantes logran usos eficientes de las herramientas y los entornos virtuales. El objetivo de este trabajo es conocer el nivel de conocimiento y las percepciones demostradas por los estudiantes respecto al uso de los entornos virtuales de aprendizaje, específicamente el Aula Virtual Moodle de la UNP; así mismo, se busca caracterizar el acceso a las condiciones de uso de la red.

Este trabajo contribuye a desarrollar mejoras desde los ámbitos de la gestión académica y administrativa de todas las unidades académicas de la UNP. Constituye un referente importante de información para que docentes, 
estudiantes y responsables de la gestión institucional articulen acciones para mejorar el uso efectivo de estos entornos, si se considera lo planteado por Osorio (2018): "mediante la investigación es posible identificar los problemas pedagógicos que se presentan en el aula y definir los correctivos necesarios para mejorar la enseñanza, como también realizar el seguimiento y evaluación de los procesos y actividades que se implementan". (p. 5)

Hoy día es impensable realizar actividades aisladas o descontextualizadas; por ello, el resultado de esta investigación permitirá la actividad conjunta de los responsables de la gestión académica y administrativa de la institución, como también la integración de saberes tecnológicos provenientes de los docentes, los técnicos y los estudiantes, de modo que se puedan aprovechar para optimizar la implementación de esta nueva modalidad de aprendizaje que constituye la inserción vertiginosa de las TIC en el ámbito de la educación superior.

Ningún sector de la educación puede evadir esta responsabilidad de actualizarse en el uso de estas tecnologías. Existen tres aspectos fundamentales para el desarrollo de la enseñanza y el aprendizaje por medio de estos entornos. El primero es la competencia digital del docente y su actitud hacia este proceso; el segundo, el dominio que pueda tener el estudiante en el manejo de entornos propicios para adquirir conocimientos, y por último la conectividad. Estos requerimientos, son muy difíciles de lograr, ya que existe una brecha digital amplia entre los sectores rurales y urbanos.

Conforme a lo expuesto por Decoud (2020), los recursos necesarios para el cumplimiento de estas políticas establecidas son muy limitados, ya que en el propio diagnóstico del Ministerio de Educación y Ciencias en 2020 se evidencia que "las instituciones educativas que cuentan con computadoras no superan el 7\% y las que están conectadas a internet representan menos del 4\%". (Decoud, 2020, p. 9)

Las competencias digitales son el conjunto de conocimientos y habilidades que permiten el uso seguro y eficiente de las TIC (Navarro y Barrios, 2016). Los requerimientos urgentes comprenden las competencias digitales de los estudiantes universitarios, los esfuerzos por minimizar las barreras sociales que incluyen la falta de habilidades computacionales básicas, la disponibilidad de la banda ancha para el acceso a internet y la falta de recursos para invertir en hardware y software. "En algunos países de Latinoamérica y el Caribe no existen o son insuficientes y dejan a las y los estudiantes en una posición de vulnerabilidad". (Decoud, 2020, p. 15)

Estas reflexiones implican el esfuerzo por parte de las instituciones para coadyuvar a la implementación efectiva de las TIC en el aprendizaje efectivo de los estudiantes. Existe un puente digital que debe ser sustentado para 
avanzar hacia la reducción de la brecha digital en América Latina y el Caribe, y los pilares del puente lo constituyen la formación y la infraestructura (Ministerio de Ciencia y Tecnología de España, s.f.). Esta formación implica la dotación de competencias para todos los involucrados en este proceso: docentes, estudiantes, funcionarios, directivos, en fin, todos los que de una u otra forma intervienen en el desarrollo o la implementación del uso de las tecnologías de la información para la formación profesional.

Por otro lado, existe una dotación de infraestructura amigable y pertinente para el uso regular y continuo de estos entornos. Otrora, la utilización de estos entornos constituía una opción, hoy día constituye un reto, una necesidad, una exigencia para el ámbito educativo. Lo importante en este proceso es considerar las motivaciones de los estudiantes, lo que implica poner en relieve las competencias del buen docente, quien deberá sortear las estrategias más pertinentes para favorecer el interés del estudiante para ampliar sus conocimientos, mediante los entornos virtuales con las herramientas pertinentes.

Tal como se plantea en este documento, es necesario desarrollar talleres de capacitación, tanto para los docentes como para los estudiantes, acerca del uso de estos entornos virtuales, considerando que estos procesos no formaban parte de la cotidianidad de ambos actores del proceso enseñanzaaprendizaje.

\section{Metodología}

Este trabajo se originó con intenciones de conocer el dominio que tienen los estudiantes de la Universidad Nacional de Pilar respecto al uso de los recursos tecnológicos, para la eficaz implementación del Aula Virtual Moodle. En ese sentido, considerando el alcance de los objetivos, se aborda un tipo de estudio descriptivo con enfoque cuantitativo y de corte transversal (Hernández et al., 2014), cuyo levantamiento de datos se hizo durante los meses de mayo y junio del año 2020.

La población de estudio se constituye por 1.318 estudiantes de cuatro unidades académicas. El tamaño de la muestra quedó conformado por 347 estudiantes, extraída proporcionalmente de cada estrato considerado, con un nivel de confianza del $95 \%$ y un margen de error admitido de $5 \%$. Para determinar el tamaño de la muestra, se consideró la cantidad de estudiantes inscritos en cada facultad, y con base en ello se consiguió el porcentaje de estudiantes que formaron parte de la muestra, conforme a lo presentado en la Tabla 1. Se utilizó la app Stats (Hernández et al., 2014). 
Tabla 1. Cálculo del tamaño de la muestra - UNP

\begin{tabular}{|l|c|c|}
\hline \multicolumn{1}{|c|}{ Unidades académicas } & $\begin{array}{c}\text { Población de } \\
\text { estudliantes (N) }\end{array}$ & Muestra (n) \\
\hline $\begin{array}{l}\text { Facultad de Ciencias Agropecuarias } \\
\text { y Desarrollo Rural }\end{array}$ & 122 & 32 \\
\hline Facultad de Ciencias Biomédicas & 100 & 26 \\
\hline $\begin{array}{l}\text { Facultad de Humanidades y Ciencias } \\
\text { de la Educación }\end{array}$ & 280 & 74 \\
\hline $\begin{array}{l}\text { Facultad de Ciencias Contables, } \\
\text { Administrativas y Económicas }\end{array} \quad$ Total & $\mathbf{N = 1 3 1 8}$ & $\mathbf{n = 3 4 7}$ \\
\hline
\end{tabular}

Fuente: elaboración propia con base en datos de la secretaría de cada unidad académica, aplicando Stats.

La técnica empleada para el levantamiento de los datos fue la encuesta que se implementó mediante un cuestionario administrado por la vía de formulario Google Forms; en este se consideraron las variables que se relacionan con el estudio, tales como: el año cursado, la percepción que tienen acerca de la implementación del aula virtual, las condiciones técnicas y cognitivas que poseen para que esta modalidad de enseñanza sea amigable y, por último, las principales dificultades que se han presentado en este proceso. El envío del formulario quedó supeditado al acceso por medio del contacto por el celular, hasta conseguir la cantidad de estudiantes requerida por cada estrato.

Una vez obtenidos los datos, se procedió al análisis de los gráficos presentados por cada variable $y$, en algunas variables muy relacionadas, se realizó un cruce de datos, conforme al porcentaje de respuestas encontradas para cada pregunta. Este análisis se realizó considerando las teorías referidas al uso adecuado de las NTIC en este momento coyuntural presentado, el cual permitió una mirada holística respecto a la situación socioeconómica y de acceso a la red, que ha determinado las brechas entre los que pudieron actualizarse con esta nueva metodología de aprendizaje, y aquellos que no han podido.

\section{Resultados y discusión}

Conforme a la metodología planteada, se pudieron sistematizar 347 respuestas de los estudiantes, que se presentan a continuación. Los estudiantes que formaron parte de la encuesta provienen de ocho carreras de las cuatro unidades académicas mencionadas de la Universidad Nacional de Pilar; la mayoría de los estudiantes son de la carrera de Licenciatura en Administración de Empresas, seguido por estudiantes de licenciatura 
en Psicología, Contaduría Pública Nacional, Ciencias de la Educación, Ingeniería Agropecuaria, Licenciatura en Enfermería, entre otros. Participaron estudiantes del primero al quinto curso de las carreras, en su mayoría del primer curso.

Figura 1. Valoración de la implementación de las clases virtuales

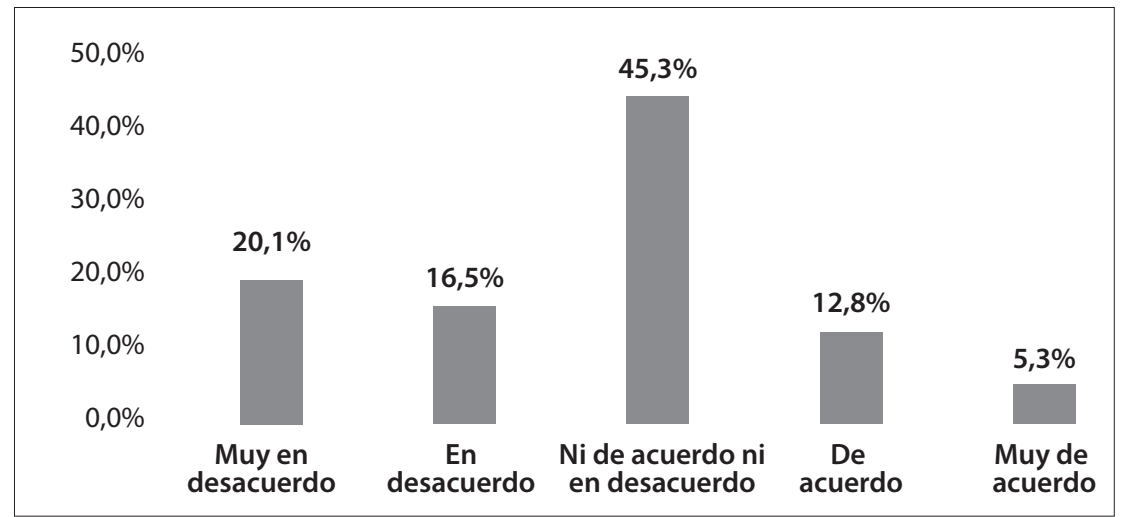

Fuente: elaboración propia con base en cuestionario aplicado a estudiantes

En esta Figura 1 se busca definir la valoración que los estudiantes manifestaron sobre este proceso de implementación de clases virtuales. Puede notarse que un elevado porcentaje manifiesta indiferencia respecto a esta implementación, el 37\% manifiesta estar entre muy en desacuerdo y en desacuerdo y tan solo un $18 \%$ dice estar entre de acuerdo y muy de acuerdo, con la implementación de esta modalidad virtual de desarrollo de las clases.

Figura 2. Tipo de celular que posee

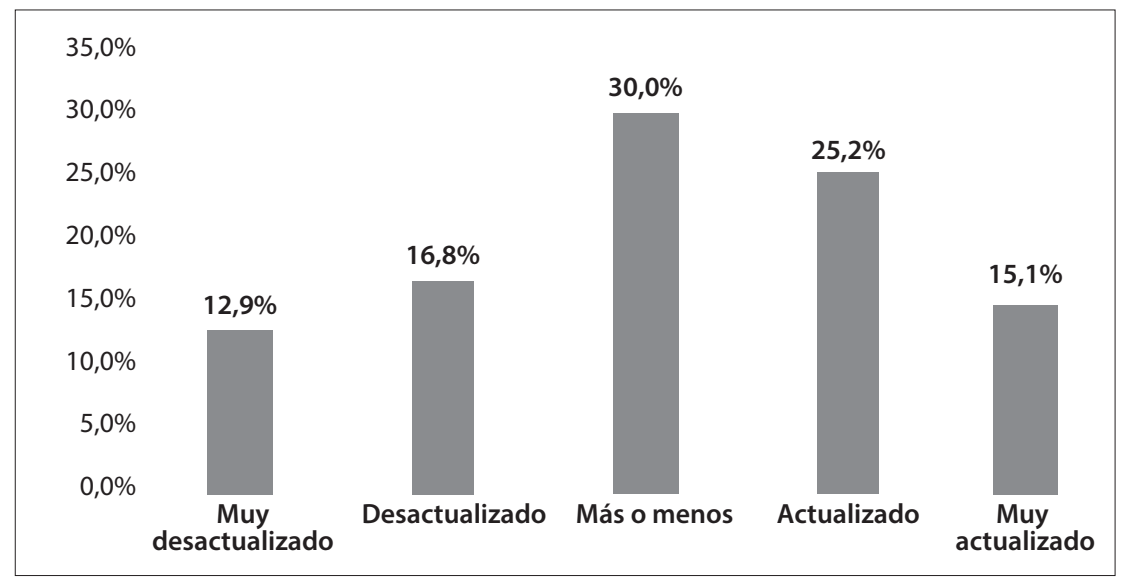

Fuente: elaboración propia con base en cuestionario aplicado a estudiantes 
La Figura 2 muestra que aproximadamente el 30\% de los estudiantes manifestaron que su celular es más o menos actualizado, el $30 \%$ que es desactualizado y muy desactualizado, y el $40 \%$ mencionaron que su celular es actualizado y muy actualizado.

Una de las condiciones indispensables para la implementación efectiva de las clases virtuales es la posesión de un aparato celular o computador que reúna las características para la utilización de las aplicaciones de los entornos virtuales, de manera amigable; en ese sentido, conforme lo plantean Navarro y Barrios (2016), la inserción al mundo digital como estrategia de aprendizaje implica la dotación de condiciones fundamentales, entre estas la dotación de una herramienta tecnológica asequible.

Figura 3. Dominio de las aplicaciones académicas

\begin{tabular}{|c|c|c|c|c|c|}
\hline $40,0 \%$ & & & $36,0 \%$ & \multirow{3}{*}{$27,7 \%$} & \\
\hline $35,0 \%$ & & & & & \\
\hline $30,0 \%$ & & & & & \\
\hline $25,0 \%$ & & & & & \\
\hline $20,0 \%$ & & $16,8 \%$ & & & \\
\hline $15,0 \%$ & & & & & \\
\hline $10,0 \%$ & $9,8 \%$ & & & & $9,8 \%$ \\
\hline $5,0 \%$ & & & & & \\
\hline $0,0 \%$ & Muy bajo & Bajo & $\begin{array}{c}\text { Ni bajo, } \\
\text { ni alto }\end{array}$ & Elevado & Muy elevado \\
\hline
\end{tabular}

Fuente: elaboración propia con base en cuestionario aplicado a estudiantes

Puede verse en la Figura 3 que aproximadamente el $40 \%$ de los estudiantes encuestados manifiestan poseer un dominio elevado y muy elevado respecto a las aplicaciones académicas, un $36 \%$ dice que lo maneja más o menos, cerca del $27 \%$ dice que tiene un dominio entre bajo y muy bajo de las mismas. El nivel de dominio favorece que los estudiantes coadyuven con el proceso de implementación de las estrategias.

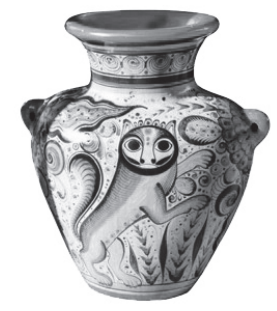


Figura 4. Condiciones de elementos requeridos y asesoramiento

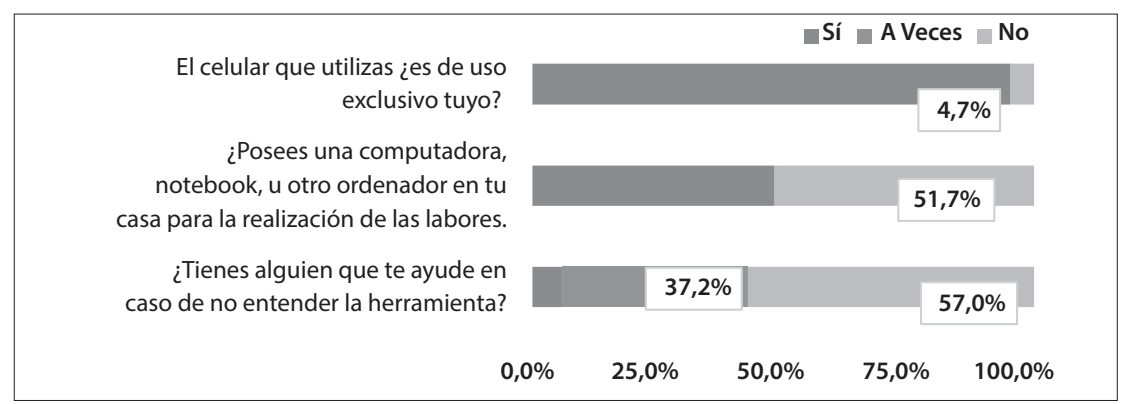

Fuente: elaboración propia con base en cuestionario aplicado a estudiantes

La Figura 4 se refiere a si el celular es de uso exclusivo del estudiante, y con sorpresa vemos que casi un $5 \%$ dice que este no es de uso exclusivo suyo; en cuanto si posee un ordenador o notebook, vimos que más del $50 \%$ no lo posee. En tanto que si son asesorados, un $60 \%$ dice que no recibe asesoría para el uso de las herramientas, un $37 \%$ dice que a veces la recibe y tan solo cerca del $6 \%$ dice recibirla.

La ventaja es que casi todos poseen un celular de uso exclusivo; no obstante, al no poseer un computador, se dificulta el uso de algunas aplicaciones y tareas que deberán ser respondidas en ese tipo de dispositivo, pues en el celular no resulta tan práctico. En cuanto al asesoramiento, es necesario que se realicen con mayor sistematicidad para aquellos estudiantes que lo necesitan, para facilitar el uso fluido del aula. Estos aspectos son los condicionantes necesarios para un buen desarrollo de las clases virtuales, conforme a los teóricos que discuten el tema de la implementación de las TIC en el aula.

Figura 5. Prerrequisitos que favorecen la utilización del aula virtual

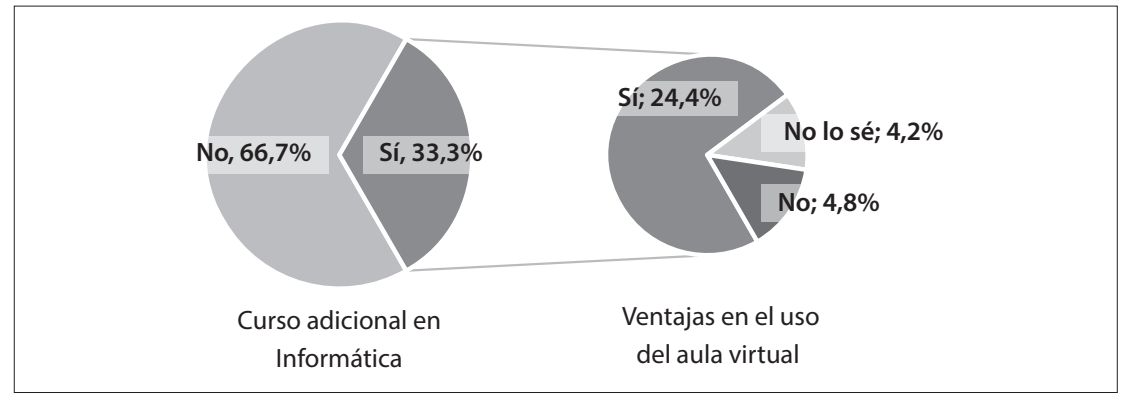

Fuente: elaboración propia con base en cuestionario aplicado a estudiantes

La Figura 5 indica si los estudiantes han hecho algún curso adicional de informática, y vemos que tan solo el $33 \%$ respondieron que sí lo hicieron y más del $66 \%$ que no; asimismo, ante la consulta si ese curso favoreció la utilización del aula virtual, el $24 \%$ de los estudiantes dijeron que sí les favoreció, 4,2\% 
dijeron no saberlo y aproximadamente $5 \%$ dijeron que no les sirvió dicho adiestramiento. Conforme a estos resultados, podemos verificar que un escaso porcentaje de estudiantes tienen una base en la utilización de entornos virtuales, la que favorecería el ingreso y desenvolvimiento en el aula virtual.

Figura 6. Nivel de la señal de Internet; Comodidad del teléfono

\begin{tabular}{|c|c|c|c|c|c|c|}
\hline \multirow[t]{2}{*}{ Señal } & \multicolumn{6}{|c|}{ Comodidad con el teléfono } \\
\hline & $\begin{array}{c}\text { Muy } \\
\text { incómodo }\end{array}$ & Incómodo & Indiferente & Cómodo & $\begin{array}{c}\text { Muy } \\
\text { cómodo }\end{array}$ & \\
\hline Muy baja & $12,3 \%$ & $7,8 \%$ & $4,2 \%$ & . $0,6 \%$ & - $1,1 \%$ & $26,1 \%$ \\
\hline Baja & $6,2 \%$ & $11,8 \%$ & $9,8 \%$ & $2,2 \%$ & - $1,1 \%$ & $31,1 \%$ \\
\hline Ni baja, ni buena & $3,4 \%$ & $3,9 \%$ & $12,6 \%$ & $5,9 \%$ & $3,6 \%$ & $29,4 \%$ \\
\hline Muy buena & $0,8 \%$ & $0,6 \%$ & $2,8 \%$ & $4,8 \%$ & $2,0 \%$ & $10,9 \%$ \\
\hline Óptima & - $0,6 \%$ & . $0,3 \%$ & . $0,3 \%$ & . $0,3 \%$ & - $1,1 \%$ & - $2,5 \%$ \\
\hline & $23,2 \%$ & $24,4 \%$ & $29,7 \%$ & $13,7 \%$ & $9,0 \%$ & \\
\hline
\end{tabular}

Fuente: elaboración propia con base en cuestionario aplicado a estudiantes

Al observar la Figura 6, de doble entrada, podemos visualizar que el 57\% de los encuestados manifiestan que la señal de internet es baja y muy baja, así como más del $47 \%$ manifiestan que su teléfono resulta muy incómodo e incómodo; aproximadamente el $30 \%$ dice que la señal no resulta tan mala ni tan buena y poco más del $13 \%$ afirman que la señal es muy buena; en cuanto a la comodidad del celular, más del $22 \%$ considera que está entre lo cómodo y muy cómodo.

Estos resultados implican que las condiciones relacionadas con la señal captada de los servicios de telefonía no son tan favorables, como tampoco la comodidad que sienten los estudiantes al utilizar su aparato celular; se debe considerar que estos aspectos son muy sensibles para los estudiantes a la hora de recibir y enviar los trabajos solicitados por los docentes. Según lo planteado por Lanuza et al. (2018), la convergencia de varios procesos y tecnologías inciden en el proceso de enseñanza-aprendizaje, pues son consideraciones que determinan el éxito o el fracaso de la implementación de estos entornos virtuales, para seguir aprendiendo.

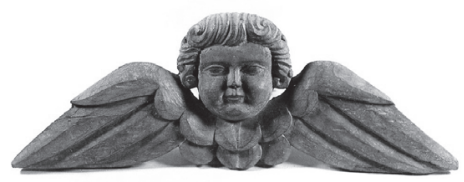


Figura 7. Provisión de la red de conexión y situación económica

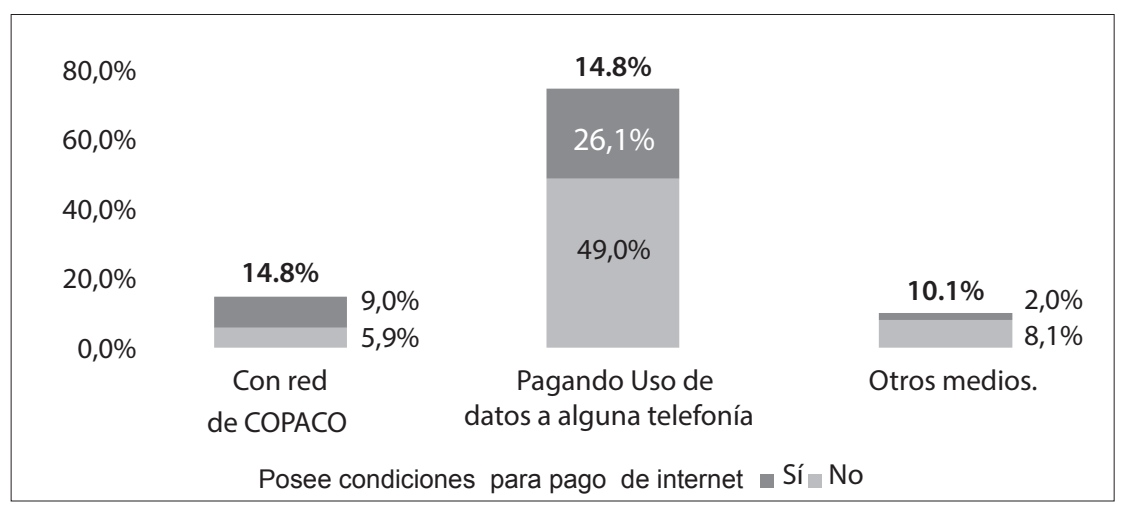

Fuente: elaboración propia con base en cuestionario aplicado a estudiantes

La barra más elevada de la Figura 7 muestra que la mayoría de los estudiantes deben conectarse a internet mediante uso de datos pagando algún servicio de telefonía, un 49\% manifiesta que económicamente no se encuentran en condiciones de hacerlo y tan solo un $15 \%$ dice que usa la red de la Compañía Paraguaya de Comunicaciones (COPACO), que constituye el uso más asequible por ser de uso familiar. Este resultado revela la dificultad manifestada por los estudiantes para el acceso al aula virtual; no obstante, sabemos que igualmente los estudiantes tratan de ponerse al día con sus actividades académicas. Este es un condicionante fundamental en este proceso, conforme lo planteado por Decoud (2020); para evitar que la brecha tecnológica aumente el rezago educativo, es imprescindible que se continúe con los proyectos de inclusión tecnológica, pues de lo contrario la brecha digital irá en aumento, dejando a estudiantes de algunos países de Latinoamérica y el Caribe en una posición de vulnerabilidad que irá desplazándolos de las posibilidades de formación profesional.

Figura 8. Acceso al Aula virtual MOODLE de la UNP

\begin{tabular}{|c|c|c|c|}
\hline $60,0 \%$ & $50,3 \%$ & \multirow[b]{2}{*}{$43,8 \%$} & \\
\hline $50,0 \%$ & & & \\
\hline $40,0 \%$ & & & \\
\hline $30,0 \%$ & & & \\
\hline $20,0 \%$ & & & \\
\hline $10,0 \%$ & & & $5,9 \%$ \\
\hline \multirow[t]{2}{*}{$0,0 \%$} & & & \\
\hline & con facilidad & con dificultad & no lo accedo \\
\hline
\end{tabular}

Fuente: elaboración propia con base en cuestionario aplicado a estudiantes 
En esta figura puede verse que más del $50 \%$ de los estudiantes acceden con facilidad al aula virtual; sin embargo, un importante $47 \%$ dice que lo accede con dificultad o no lo accede. Esto llama la atención y permite deducir que nuestros alumnos no están tan preparados para trabajar por estas vías. A esta realidad se suma que, conforme a la Figura 9 , un $85 \%$ dice que nunca han trabajado de esta manera durante su curso. Estos hallazgos permiten reflexionar acerca de lo que Cabrol (2019) plantea en términos de que en educación debemos modificar la manera en que nos acercaremos a los estudiantes.

Las nuevas metodologías pedagógicas y el uso de la tecnología requieren que el sistema educativo y los profesores se aproximen de forma distinta a la educación, eso implica la adquisición de competencias digitales fundamentales y conlleva a la necesidad de prepararse impetuosamente para esta sociedad tecnológica, por la inserción sin retorno a esta modalidad en todos los ámbitos del quehacer humano, y más aún en el ámbito educativo.

Figura 9. Habilidades de estudio independiente

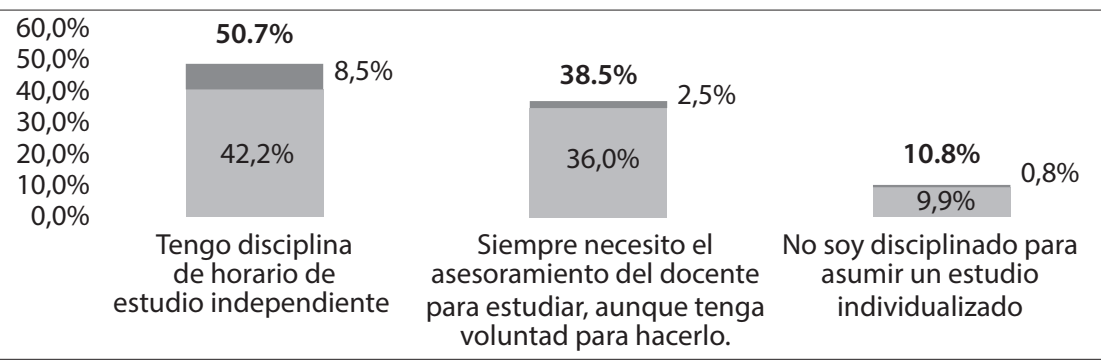

Fuente: elaboración propia con base en cuestionario aplicado a estudiantes

Esta gráfica señala que, entre las habilidades de estudio independiente, más del $50 \%$ de los estudiantes dicen tener disciplina en sus horarios de estudios; sin embargo, el 38,50\% dice que siempre necesita el asesoramiento del docente y el 10,73\% de los encuestados afirmaron no poseer disciplina para asumir un estudio individualizado. Ante la pregunta de si han participado en cursos desarrollados con entornos virtuales, se observa que más del $80 \%$ respondió que no. Es fundamental la autodisciplina para que los estudiantes puedan ponerse al día con las actividades solicitadas en este proceso.

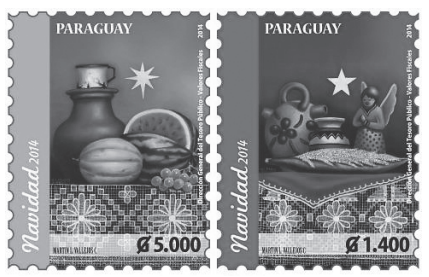


Figura 10. Dificultades avizoradas en este proceso

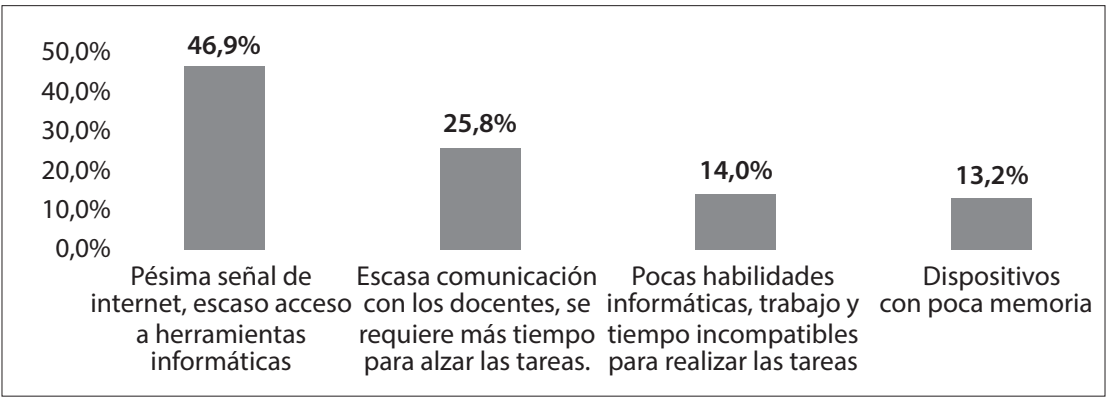

Fuente: elaboración propia con base en cuestionario aplicado a estudiantes

En la Figura 10 se observan las dificultades más repetidas, que fueron: la poca conectividad a internet, la escasa comunicación con los docentes, la poca habilidad para el uso de la tecnología, los dispositivos con poca memoria y otras que se mencionan a continuación tal como fueron expresadas por los encuestados:

- No tengo una notebook y me dificulta hacer algunos trabajos en el celular.

- Atender a todas las fechas de las tareas. Y algunos profesores alzan la tarea con pocos días de aviso, lo que dificulta organizar mis tiempos para cumplir todas mis responsabilidades.

- Pésima señal de las telefonías, y tanto la facultad como los docentes ven esto como una manera de salvar el momento en vez de hacerlo bien. Las 'clases' que realizan los docentes no están diseñadas para hacerlo de forma virtual, porque piensan que lo hacen de forma presencial y son muy largas, las clases online tienen que ser concisas y rápidas no deberían de llevar más de 40 minutos y tampoco se debería de estar leyendo los archivos pdf; para eso deben enviar antes y las dudas son las que deben ser tratadas en las clases, lo cual son pocos los docentes que lo hacen.

- Realmente es complicado. A mí me causa mucho estrés y el hecho de no entender varias cosas me desespera, me gustaría que volvamos a un aula, se me da mejor estudiar así, tal vez deje este año la facultad y vuelva el próximo porque es mejor para mí. Falta de tiempo porque trabajo, entre otras cosas.

Estos resultados revelan coincidencias con lo planteado por el Ministerio de Ciencia y Tecnología de España (s.f.), que menciona que existe un puente digital que debe ser sustentado para avanzar hacia la reducción de la brecha digital en América Latina y el Caribe; es bueno insistir en que los pilares del puente lo constituyen la formación tanto de los docentes como de los alumnos y la infraestructura. 
Figura 11. Algún valor avizorado en este proceso

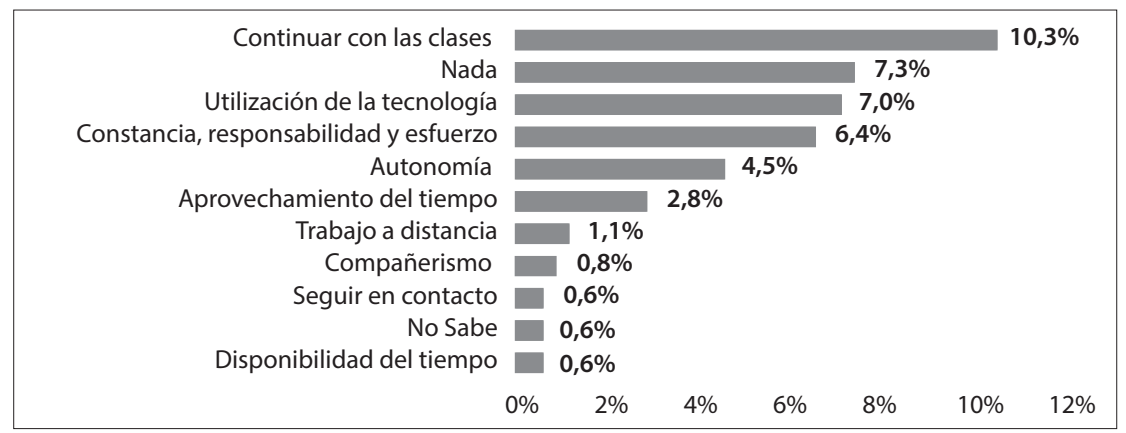

Fuente: elaboración propia con base en cuestionario aplicado a estudiantes

El $45 \%$ de los estudiantes por sobre todo valoran el hecho de que no perderán el año lectivo, el $20 \%$ dijo que no existe ningún valor que pueda rescatar de este proceso; otros, sin embargo, valoraron la independencia, la autodeterminación, la responsabilidad, el autodidactismo, la paciencia, la perseverancia, entre otros aspectos positivos que le encontraron a este proceso. A continuación, se enlistan algunas respuestas dadas por los estudiantes consultados respecto al valor avizorado en este proceso:

- Permite seguir la formación de los alumnos de una manera diferente y así aprender nuevas cosas y adquirir esa experiencia.

- Me parece una buena alternativa, lástima que no puedo acceder bien por la poca señal en la zona y por el dispositivo con poca memoria.

- Que nos apoyamos entre todos y siempre teniendo la voluntad de ayudar al que no puede.

- Permite seguir la formación del alumno de una manera diferente y así aprender nuevas cosas y adquirir esa experiencia.

\section{Conclusiones}

Al analizar los resultados de este cuestionario, se puede concluir que los estudiantes, en su mayoría, están de acuerdo con la utilización el aula virtual para salvar esta situación generada a causa de la pandemia. No obstante, es necesario revisar aspectos relacionados con lo económico en lo que respecta al acceso de las redes y la internet gratuita. Hasta este momento, en términos generales, para los alumnos se constituye más bien en una debilidad, dada la incursión abrupta al aula virtual. A ellos les surgen inconvenientes porque no han interactuado anteriormente en este tipo de entorno de aprendizaje, por lo que esa migración gradual no fue entrenada sino instaurada de manera brusca. 
Para mejorar este proceso, cabe reflexionar sobre distintos aspectos descritos por los estudiantes y que recogemos a continuación:

- Comunicación más directa por parte de los docentes con los alumnos.

- Situación socioeconómica que limita la compra de datos para uso de internet.

- Elaboración de guías didácticas pertinentes y de fácil comprensión para los alumnos.

- Baja conectividad de los servicios de telefonía.

- Utilización más frecuente de videoconferencias explicativas e interactivas con los docentes.

- Escaso desarrollo de estudio independiente por parte de los estudiantes.

El resultado de esta investigación se ha presentado a las instancias misionales y de gestión de la Universidad Nacional de Pilar, con el propósito de tomar las medidas correctivas para que se establezcan las mejoras necesarias, tales como capacitación a los docentes y los alumnos, y así optimizar los resultados esperados mediante la implementación del aula virtual. Se presentan la discusión y los resultados mediante un artículo científico para que sean difundidos y transferidos a la comunidad, de tal modo que los estamentos gubernamentales y municipales dimensionen la necesidad de apoyar al Sistema Educativo Nacional.

$$
* * *
$$

\section{Referencias bibliográficas}

1. Benítez, G. (2007). NTICs, Interacción y Aprendizaje en la Universidad. [Tesis de doctorado, Universitat Rovira I Virgili]. https://www.tdx.cat/bitstream/ handle/10803/8929/portadaindice.pdf?sequence=36\&isAllowed=y

2. Cabrol, M. (2019). Nuevas formas de enseñar y de aprender. En Mateo, M. y Rucci, G. El Futuro ya está aquí. Habilidades transversales en América Latina y el Caribe, en el siglo XXI (107-220). Banco Internamericano de Desarrollo. https://publications.iadb.org/es/el-futuro-ya-est\%C3\%A1-aqui-habilidadestransversales-de-america-latina-y-el-caribe-en-el-siglo-xxi

3. Consejo Nacional de Educación Superior. (2020). CONES. Gobierno Nacional de Paraguay. http://www.cones.gov.py/educacion-superior-del-paraguayen-tiempos-de-covid-19/ 
4. Decoud, C. (2020). Uso de las Tic en el aula con enfoque CTS. Consejo Nacional de Ciencia y Tecnología. https://cts.conacyt.gov.py/course/index. php?categoryid $=26$

5. Hernández, R., Fernández, C. y Baptista, P. (2014). Metodología de la Investigación (6 ed.). McGraw-Hill/Interamericana Editores, S.A.

6. Lanuza, F., Rizo, M. y Saavedra, L. (2018). Uso y aplicación de las TIC en el proceso de enseñanza-aprendizaje. Revista Científica de FAREM-Estelí. Medio ambiente, tecnología y desarrollo humano, (25), 16-30. https://doi. org/10.5377/farem.v0i25.5667

7. Ministerio de Ciencia y Tecnología de España. (s.f.). Sociedad de la Información en el siglo XXI: un requisito para el desarrollo. Buenas prácticas y lecciones aprendidas. Gobierno de España. https://www.itu.int/net/wsis/stocktaking/ docs/activities/1103547250/sociedad-informacion-sigloxxi-es.pdf

8. Navarro, R. y Barrios, S. (2016). Las competencias digitales en la educación superior. EduDoc - Centro de documentación sobre educación. http://quijote. biblio.iteso.mx/catia/edudocdc/cat.aspx?cmn=download\&ID=328\&N=1

9. Organización de las Naciones Unidas para la Educación, la Ciencia y la Cultura - UNESCO. (2013). Enfoques estratégicos de las TICS sobre Educación en América Latina y el Caribe. OREAL/UNESCO. http://www.unesco.org/new/ fileadmin/MULTIMEDIA/FIELD/Santiago/images/ticsesp.pdf

10. Osorio M., C. A. (2018). La investigación interdisciplinaria: la enseñanza por proyectos. Consejo Nacional de Ciencia y Tecnología - CONACYT, Gobierno de Paraguay.

Para citar este artículo:
Velázquez, D. y Torres V., N. (2021). Conocimiento y percepciones de los estudiantes del aula virtual en la Universidad Nacional de Pilar. Teuken Bidikay, 12(18), 219-234 doi: 10.33571/teuken.v12n18a11

Ge*: AMV.

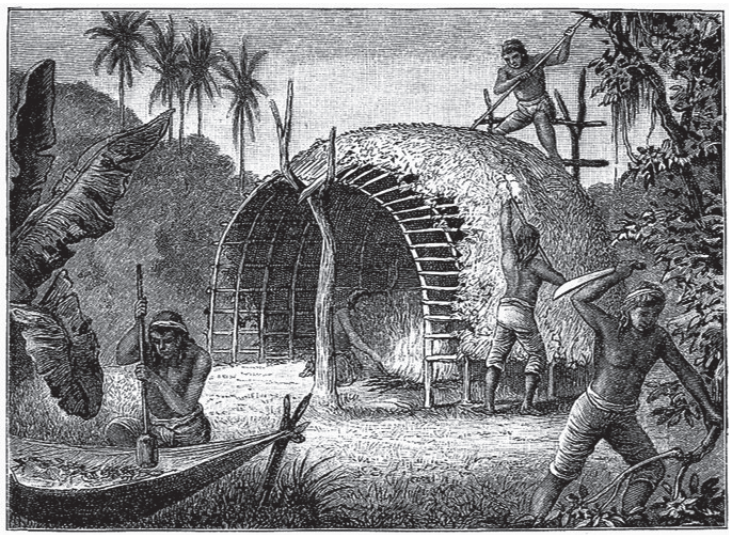

